\title{
Sequential quantum-enhanced measurement with an atomic ensemble
}

\author{
A. V. Lebedev, ${ }^{1, *}$ P. Treutlein, ${ }^{2}$ and G. Blatter ${ }^{1}$ \\ ${ }^{1}$ Theoretische Physik, Wolfgang-Pauli-Strasse 27, ETH Zurich, CH-8093 Zürich, Switzerland \\ ${ }^{2}$ Department of Physics, University of Basel, Klingelbergstrasse 82, CH-4056 Basel, Switzerland
}

(Received 17 April 2013; published 21 January 2014)

\begin{abstract}
We propose a quantum-enhanced iterative (with $K$ steps) measurement scheme based on an ensemble of $N$ two-level probes which asymptotically approaches the Heisenberg limit $\delta_{K} \propto R^{-K /(K+1)}$, where $R$ is the number of quantum resources. The protocol is inspired by Kitaev's phase estimation algorithm and involves only collective manipulation and measurement of the ensemble. The iterative procedure takes the shot-noise-limited primary measurement with precision $\delta_{1} \propto N^{-1 / 2}$ to increasingly precise results, $\delta_{K} \propto N^{-K / 2}$. We propose an implementation of the algorithm for the measurement of a magnetic field using a two-component atomic cloud of bosons.
\end{abstract}

DOI: 10.1103/PhysRevA.89.012118

PACS number(s): 03.65.Ta, 03.67.Ac, 06.20.-f

Introduction. Estimating an unknown parameter $\phi$ of a quantum system usually involves a quantum probe prepared in a known state $\hat{\rho}_{0}$ which, when brought into interaction with the system, evolves to a new state $\hat{\rho}_{\phi}$ under the action of a (so-called) quantum channel [1] $\mathcal{Q}_{\phi}, \hat{\rho}_{0} \rightarrow \mathcal{Q}_{\phi}\left(\hat{\rho}_{0}\right)=\hat{\rho}_{\phi}$; by measuring a suitable observable of the probe in state $\hat{\rho}_{\phi}$, one can infer the value of $\phi$. Given the fundamental uncertainty of the quantum measurement, this information has a statistical character. In order to improve the knowledge of $\phi$, one needs to repeat the measurement: using $R \gg 1$ independent quantum probes $\hat{\rho}_{0}^{\otimes R} \rightarrow\left[\mathcal{Q}_{\phi}\left(\hat{\rho}_{0}\right)\right]^{\otimes R}=\hat{\rho}_{\phi}^{\otimes R}$ leads to a $\sqrt{R}$ increase in the measurement precision $\delta_{0} \rightarrow \delta_{0} / \sqrt{R}$ : this is the standard quantum or shot-noise limit of measurement. Incidentally, nature provides us with a better, albeit ultimate [2], Heisenberg limit, $\delta_{0} \rightarrow \delta_{0} / R$, if one exploits some of the quantum resources of the system under investigation. Exploiting such quantum effects enhancing the measurement precision is the subject of quantum metrology $[1,3]$.

The quantum enhancement in the measurement precision can be approached by using either parallel or sequential strategies [4]. In a parallel strategy, the original ensemble is divided into $m$ subensembles with $n$ probes, $R=n m$, with each subensemble prepared in a (maximally) entangled state, $\left[\hat{\rho}_{0}^{(n)}\right]^{\otimes m} \rightarrow\left[\mathcal{Q}_{\phi}\left(\hat{\rho}_{0}^{(n)}\right)\right]^{\otimes m}$; this results (ideally) in a $\sqrt{n}$ enhancement of the precision compared to the standard quantum limit, $\delta_{0} \rightarrow \delta_{0} / \sqrt{m} n=\delta_{0} \sqrt{m} / R$ (see Refs. [5] for the case of quantum interferometry and Refs. [6] for a general quantum channel). Alternatively, in a sequential strategy, instead of preparing entangled states, each of $m$ separate probes is passed $n$ times through the same quantum channel, $\hat{\rho}_{0}^{\otimes m} \rightarrow\left[\mathcal{Q}_{\phi}^{n}\left(\hat{\rho}_{0}\right)\right]^{\otimes m}$, resulting in the same enhancement in precision [see Refs. [7]]. Roughly speaking, while parallel strategies make use of entanglement, sequential strategies exploit the coherent quantum dynamics as a resource in order to enhance the measurement precision. While sequential strategies do not require the creation of rather fragile entangled states, they do demand longer (coherence) times to allow for completion of the measurement.

\footnotetext{
*lebedev@itp.phys.ethz.ch
}

Recently, quantum measurement protocols were implemented using two-component atomic ensembles with $N$ particles [8-10]. In Ref. [8], interatomic interactions were used to create entangled, spin-squeezed states [11] of BoseEinstein condensates that allow one to go beyond the standard quantum limit via a parallel strategy. Here, we suggest a sequential strategy without entanglement, allowing us to reach a given precision $\delta$ within $K \sim \ln \left(\delta_{1} / \delta\right) / \ln (\sqrt{N})$ steps, where $\delta_{1}=\delta_{0} / \sqrt{N}$ is the uncertainty of the primary measurement and $N \gg 1$ is the size of the ensemble. This requires an $\sim \sqrt{N}$-fold longer evolution under the unknown external field in each subsequent measurement step. Our approach resembles Kitaev's phase estimation algorithm [12] as well as algorithms [13-15] based on the semiclassical Fourier transform [16]; such algorithms, making use of individual qubits, have been successfully implemented recently [17]. Exploiting the large ensemble of $N$ individual probes, our scheme requires far fewer steps to complete the measurement; furthermore, it does not require separate access to the individual probes in the ensemble and relies only on collective manipulations and measurements.

Primary measurement. We wish to estimate the real angle (or phase [18]) $\phi \in[-\pi, \pi]$ in an unknown unitary rotation $\hat{U}_{z}[\phi]=\exp \left[-i \hat{\sigma}_{z} \phi / 2\right]$ given an ensemble (with $N \gg 1$ probes) of spin-1/2 systems (qubits) and using only collective unitary operations over the ensemble. We use Ramsey interferometry as the primary measurement and prepare all qubits in the $\hat{\sigma}_{x}=1$ polarized state $(|\uparrow\rangle+$ $|\downarrow\rangle) / \sqrt{2}$. Applying $\hat{U}_{z}[\phi]$ to the ensemble results in the state $\left[\left(e^{-i \phi / 2}|\uparrow\rangle+e^{+i \phi / 2}|\downarrow\rangle\right) / \sqrt{2}\right]^{\otimes N}$. A $\hat{U}_{y}[-\pi / 2]$ pulse rotates the ensemble into the readout state $[\cos (\phi / 2)|\uparrow\rangle+$ $i \sin (\phi / 2)|\downarrow\rangle]^{\otimes N}$, and measuring the total polarization $\hat{S}_{z}=$ $(1 / N) \sum_{i=1}^{N} \hat{\sigma}_{z}^{(i)}$, one arrives at one of the possible outcomes $S_{z}=\left[N_{+}-N_{-}\right] / N$, with $N_{+} \in\{0, \ldots, N\}$ and $N_{-}=N-$ $N_{+}$the number of qubits observed in the $\sigma_{z}= \pm 1$ states. The probability of observing a particular value $\tilde{S}_{z}$ (we denote by $\tilde{X}$ a realized value of the random variable $X$ ) is given by the Bernoulli distribution,

$$
\mathcal{P}\left(S_{z}=\tilde{S}_{z} \mid \phi\right)=\frac{N !}{\tilde{N}_{+} ! \tilde{N}_{-} !}\left(\cos ^{2} \frac{\phi}{2}\right)^{\tilde{N}_{+}}\left(\sin ^{2} \frac{\phi}{2}\right)^{\tilde{N}_{-}}
$$


conditioned on the unknown value of $\phi$. Starting with an unbiased, homogeneous distribution $P(\phi)$ for the parameter $\phi$ (no a priori knowledge of $\phi$ ), the measurement of a particular value of $\tilde{S}_{z}$ allows us to improve our statistical information on $\phi$. Changing variables $\phi \rightarrow p=\cos \phi$ and making use of Bayes' theorem, the a posteriori probability distribution $P\left(p \mid \tilde{S}_{z}\right)$ of $p \in[-1,1]$ knowing the measured result $\tilde{S}_{z}$ is enhanced by the factor $\mathcal{P}\left(S_{z}=\tilde{S}_{z} \mid \phi\right) / \mathcal{P}\left(S_{z}\right)$; hence, after proper normalization,

$$
P\left(p \mid \tilde{S}_{z}\right)=\frac{(N+1) !}{2 \tilde{N}_{+} ! \tilde{N}_{-} !}\left(\frac{1+p}{2}\right)^{\tilde{N}_{+}}\left(\frac{1-p}{2}\right)^{\tilde{N}_{-}} .
$$

In the following, we consider the limit of large $\tilde{N}_{+}, \tilde{N}_{-} \gg$ 1 , where the distribution $P\left(p \mid \tilde{S}_{z}\right)$ has a sharp peak near $\tilde{S}_{z}$; expanding the exponent in $\exp \left[\tilde{N}_{+} \ln (1+p)+\tilde{N}_{-}(1-p)\right]$ around the maximum, the distribution $P\left(p \mid \tilde{S}_{z}\right)$ then can be replaced by the normal distribution

$$
P\left(p \mid \tilde{S}_{z}\right)=\frac{1}{\sqrt{2 \pi} \sigma} \exp \left[-\frac{\left(p-\tilde{S}_{z}\right)^{2}}{2 \sigma^{2}}\right], \quad \sigma^{2}=\frac{1-\tilde{S}_{z}^{2}}{N},
$$

or, in another notation, $p \sim \mathcal{N}\left(\tilde{S}_{z}, \sigma^{2}\right)$. The overall result of the above ensemble measurement is summarized in the following statistical statement: Given the tolerance level $\beta \ll 1$, the precision of the estimate $p \approx \tilde{S}_{z}$ is given by

$$
\operatorname{Prob}\left[\left|p-\tilde{S}_{z}\right| \leqslant g(\beta) \sigma\right]=1-\beta,
$$

where $g$ is determined by $1-\beta=\operatorname{erf}(g / \sqrt{2})$ and $\operatorname{erf}(x)=$ $(1 / \sqrt{\pi}) \int_{-x}^{x} d t e^{-t^{2}}$ is the standard error function. Finally, distribution (3) for $p=\cos \phi$ provides us with the distribution function for the angle $\phi$ :

$$
P\left(\phi \mid \tilde{S}_{z}\right)=\frac{|\sin \phi|}{2 \sqrt{2 \pi} \sigma} \exp \left[-\frac{\left(\cos \phi-\tilde{S}_{z}\right)^{2}}{2 \sigma^{2}}\right] .
$$

Complementary measurement. Since $\cos \phi$ is even in $\phi$, the estimate for $\phi$ is distributed among two symmetric intervals, $|\phi \pm \tilde{\phi}| \leqslant g / \sqrt{N}$, with $\tilde{\phi}=\left|\arccos \left(\tilde{S}_{z}\right)\right|$. Expanding (5) near $\phi \approx \pm \tilde{\phi}$, the distribution of $\phi$ is given by the sum of two normal distributions,

$$
\phi \sim \frac{1}{2} \sum_{\alpha= \pm 1} \mathcal{N}\left(\alpha \tilde{\phi}, \sigma_{1}^{2}\right), \quad \sigma_{1}=\frac{1}{\sqrt{N}},
$$

describing two equiprobable alternatives $\alpha= \pm 1$ for the angle $\phi$ to be located near $\alpha \tilde{\phi}$. In order to distinguish between the two alternatives of the primary measurement, we perform a second test by preparing the ensemble in the $\sigma_{y}=+1$ polarized state, $([|\uparrow\rangle+i|\downarrow\rangle] / \sqrt{2})^{\otimes N^{\prime}}$. Repeating the Ramsey measurement, the second estimate for the parameter $p^{\prime}=\sin \phi$ should result in either $+\sin \tilde{\phi}$ or $-\sin \tilde{\phi}$, thus distinguishing the alternatives $+\tilde{\phi}$ and $-\tilde{\phi}$ provided by the first measurement. Specifically, given the probability of observing a qubit in the $\sigma_{z}= \pm 1$ state conditioned on the result $\alpha \tilde{\phi}$ of the primary measurement,

$$
\begin{aligned}
\mathcal{P}\left(\hat{\sigma}_{z}= \pm 1 \mid \alpha \tilde{\phi}\right) & =\frac{1}{2} \int d \phi \frac{1 \pm \sin \phi}{2} \sum_{\alpha= \pm 1} \mathcal{N}\left(\alpha \tilde{\phi}, \sigma_{1}^{2}\right) \\
& \approx\left[1 \pm\left(\alpha \sin \tilde{\phi}-\sin ^{2} \tilde{\phi} / 2 N\right)\right] / 2,
\end{aligned}
$$

we find that the total polarization of the complementary ensemble is given by the sum of two normal distributions,
$S_{z}^{\prime} \sim \sum_{\alpha} \mathcal{N}\left(S_{z \alpha}^{\prime}, \sigma^{\prime 2}\right) / 2$, with mean and variance

$$
S_{z \alpha}^{\prime} \approx \alpha \sin \tilde{\phi}-\frac{\sin ^{2} \tilde{\phi}}{2 N}, \quad \sigma^{\prime 2} \approx \frac{\cos ^{2} \tilde{\phi}}{N^{\prime}} .
$$

To construct an unbiased classification rule we define the regions $E_{-}=\left\{S_{z}^{\prime} \mid S_{z}^{\prime}<\bar{S}_{z}^{\prime}\right\}$ and $E_{+}=\left\{S_{z}^{\prime} \mid S_{z}^{\prime}>\bar{S}_{z}^{\prime}\right\}$, with the boundary $\bar{S}_{z}^{\prime}$ set by the condition

$$
\mathcal{P}\left(E_{-}\right) \equiv \int_{-\infty}^{\bar{S}_{z}^{\prime}} d S_{z}^{\prime} P\left(S_{z}^{\prime}\right)=\mathcal{P}\left(E_{+}\right)=1 / 2 .
$$

In our symmetric situation, $\bar{S}_{z}^{\prime}=\left(S_{z-}^{\prime}+S_{z+}^{\prime}\right) / 2$. Given a measured $\tilde{S}_{z}^{\prime}$ we then assign the value $\alpha=1 \quad(\alpha=-1)$ whenever the event has been realized in $E_{+}\left(E_{-}\right)$. This assignment is prone to a misclassification error, $\beta^{\prime}=$ $\mathcal{P}\left(+\mid E_{-}\right) \mathcal{P}\left(E_{-}\right)+\mathcal{P}\left(-\mid E_{+}\right) \mathcal{P}\left(E_{+}\right)$, where the conditional probability $\mathcal{P}\left(+\mid E_{-}\right)=\mathcal{P}\left(E_{-} \mid+\right) \mathcal{P}(+) / \mathcal{P}\left(E_{-}\right)$[and similarly for $\left.\mathcal{P}\left(-\mid E_{+}\right)\right]$follows from Bayes' theorem; for our unbiased classification rule, this reduces to $\mathcal{P}\left(\alpha^{\prime} \mid E_{\alpha}\right)=\mathcal{P}\left(E_{\alpha} \mid \alpha^{\prime}\right)$. The conditional probabilities $\mathcal{P}\left(E_{\alpha} \mid \alpha^{\prime}\right)$ are easily obtained from the distributions $\mathcal{P}\left(S_{z}^{\prime} \mid \alpha\right)=\mathcal{N}\left(S_{z \alpha}^{\prime}, \sigma^{\prime 2}\right)$, and we find that

$$
\beta^{\prime}=\left[1-\operatorname{erf}\left(|\sin \tilde{\phi}| / \sqrt{2} \sigma^{\prime}\right)\right] / 2 .
$$

Away from the immediate vicinity of $\tilde{\phi} \approx 0$ (e.g., $\tilde{N}_{+}>5$ ) and a typical number of probes $N \sim 10^{3}$, choosing $N^{\prime} \sim N$ results in a negligible probability $\beta^{\prime}$ of misclassification.

$\mathrm{n}$-fold rotation. Next, we analyze the sequential $(n>$ 1-fold) application of the rotation $\hat{U}_{z}[\phi]$ in the Ramsey measurement. Measuring the ensemble polarization $\hat{S}_{z}$ then provides an estimate for the parameter $\phi_{n}=n \phi$. Given a measured result $\tilde{S}_{z n}$, the a posteriori distribution function for the parameter $\phi_{n}$ is given by Eq. (5) with $\tilde{S}_{z} \rightarrow \tilde{S}_{z n}$ and $\phi \rightarrow$ $\phi_{n}$, providing two Gaussian peaks at $\tilde{\phi}_{n}= \pm\left|\arccos \left(\tilde{S}_{z n}\right)\right|$ of width $\propto 1 / \sqrt{N}$ as a function of $\phi_{n}$; the accompanying complementary measurement again selects one alternative $\alpha= \pm 1$.

A further estimate of the angle $\phi$ is obtained by reading the distribution function Eq. (5) with $\cos \phi \rightarrow \cos (n \phi)$ as a function of $\phi$. The periodicity of $\cos (n \phi)$ provides $n$ different values of $\phi$, all corresponding to the same value of $\cos (n \phi)$. The distribution function $P_{n}(\phi)$ for the angle $\phi$ then has $n$ peaks centered at $\tilde{\phi}_{n k}$,

$$
\begin{aligned}
\phi & \sim \frac{1}{n} \sum_{k=0}^{n-1} \mathcal{N}\left(\tilde{\phi}_{n k}, \sigma_{n}^{2}\right), \quad \sigma_{n}=\frac{\sigma_{1}}{n}, \\
\tilde{\phi}_{n k} & =\frac{\tilde{\phi}_{n}}{n}+2 \pi \frac{k}{n}, \quad k=0, \ldots, n-1,
\end{aligned}
$$

each peak $n$ times narrower than in the previous $n=1$ case. As a result, the $n$-fold measurement redistributes the original uncertainty $\delta_{1} \sim \sigma_{1}$ among $n$ different equiprobable positions, which we call the alternatives $A_{k}$. While this result does not give us more a posteriori information on the position of $\phi$ than the onefold measurement [as confirmed by the Shannon entropy $H_{n}=-\int d \phi P_{n}(\phi) \ln P_{n}(\phi)$ coinciding for all $n$ ], the different distribution of the probability allows us to gain in precision when combining the two measurements.

Beyond the shot-noise limit. In order to take advantage of the $n$-fold measurement one has to identify the correct alternative 


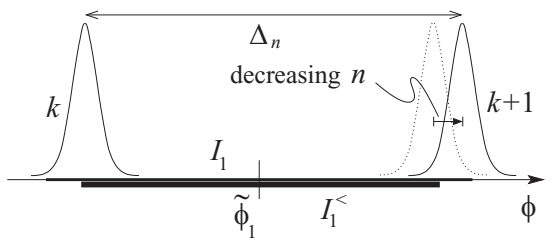

FIG. 1. Intervals $I_{1}$ and $I_{1}^{<}$centered around $\tilde{\phi}_{1}$. The peaks belonging to alternatives $A_{k}$ and $A_{k+1}$ are shown for the extreme case where $\tilde{\phi}_{n k}$ coincides with the left boundary and $\tilde{\phi}_{n k}$ is on the verge of leaving $I_{1}^{<}$as $n$ decreases, $\Delta_{n}=2 \pi / n$.

among the $n$ equiprobable distributions [see Eq. (11)]. This is done by combining the results of the $1-$ and $n$-fold measurements. We define the interval $I_{1}=\left\{\phi|| \phi-\tilde{\phi}_{1} \mid \leqslant\right.$ $\left.g(\beta) \sigma_{1}\right\}$ centered around the result $\tilde{\phi}_{1}$ of the first measurement (see Fig. 1), with $\beta$ the tolerance level of the first measurement, hence $\operatorname{Prob}\left[\left|\phi-\tilde{\phi}_{1}\right| \leqslant g(\beta) \sigma_{1}\right]=1-\beta$ [cf. Eq. (4)]. We call the alternative $A_{k}$ compatible with the first measurement if the condition

$$
\operatorname{Prob}\left[\phi \in I_{1} \mid \phi \sim \mathcal{N}\left(\tilde{\phi}_{n k}, \sigma_{n}^{2}\right)\right] \geqslant 1-\beta
$$

is satisfied. In order to satisfy the original confidence level in the second measurement, the maximum $\tilde{\phi}_{n k}$ belonging to $A_{k}$ must be located within the reduced interval $I_{1}^{<}=\{\phi|| \phi-$ $\left.\tilde{\phi}_{1} \mid \leqslant g(\beta)\left(\sigma_{1}-\sigma_{n}\right)\right\}$ (see Fig. 1).

By construction, condition (13) is satisfied for at least one $k$, irrespective of the value of $n$. Choosing a small $n$, the gain in precision is small, hence we are interested in maximizing the value of $n$. On the other hand, for large $n$, the number of peaks compatible with Eq. (13) is greater than one and we cannot select the proper alternative $A_{k}$. The optimal number $n_{\text {opt }}$ can be determined by considering the situation where the $k$ th peak is located at the left boundary of $I_{1}^{<}, \tilde{\phi}_{n k}=\tilde{\phi}_{1}-$ $g(\beta)\left(\sigma_{1}-\sigma_{n}\right)$, while the next peak, $\phi_{n k+1}$, is being pushed out from $I_{1}^{<}$across the right boundary of $I_{1}^{<}$with decreasing $n$ (see Fig. 1). Obviously, when $\tilde{\phi}_{n k+1}=\tilde{\phi}_{1}+g(\beta)\left(\sigma_{1}-\sigma_{n}\right)$ we still have two equally probable alternatives generating a large misclassification error $\tilde{\beta}=1 / 2$. The task, then, is to find the largest possible $n$ compatible with a prescribed error $\tilde{\beta} \ll 1$.

After two measurements, a 1- and an $n$-fold, the a posteriori distribution function for the angle $\phi$ is given by

$$
P\left(\phi \mid \tilde{\phi}_{1}, \tilde{\phi}_{n}\right) \propto \sum_{k=0}^{n-1} \frac{w_{k}}{\sqrt{2 \pi} \sigma_{1, n}} \exp \left[-\frac{\left(\phi-\tilde{\phi}_{1, n k}\right)^{2}}{2 \sigma_{1, n}^{2}}\right],
$$

with $\quad \tilde{\phi}_{1, n k}=\left(\tilde{\phi}_{1} \sigma_{n}^{2}+\tilde{\phi}_{n k} \sigma_{1}^{2}\right) /\left(\sigma_{1}^{2}+\sigma_{n}^{2}\right) \approx \tilde{\phi}_{n k}, \quad \sigma_{1, n}=$ $\sigma_{1} \sigma_{n} / \sqrt{\sigma_{1}^{2}+\sigma_{n}^{2}} \approx \sigma_{n}$, and $w_{k}$ is the a posteriori probability that the $k$ th alternative has been realized,

$$
w_{k}=\exp \left[-\frac{\left(\tilde{\phi}_{1}-\tilde{\phi}_{n k}\right)^{2}}{2\left(\sigma_{1}^{2}+\sigma_{n}^{2}\right)}\right] .
$$

For our arrangement $\tilde{\phi}_{n k}=\tilde{\phi}_{1}-g(\beta)\left(\sigma_{1}-\sigma_{n}\right)$ and $\tilde{\phi}_{n k+1}=$ $\tilde{\phi}_{1}-g(\beta)\left(\sigma_{1}-\sigma_{n}\right)+\Delta_{n}$. Considering only these two peaks, the misclassification error of the $k$ th alternative is given by $\tilde{\beta}=w_{k+1} /\left(w_{k}+w_{k+1}\right)$. Solving for $\Delta_{n}$, we obtain the optimal number of rotations compatible with $\tilde{\beta}, n \leqslant n_{\text {opt }}=$ $\left\lfloor v(\beta, \tilde{\beta}) / \sigma_{1}\right\rfloor \equiv n_{2}$, with

$$
v=\pi g(\beta) \frac{\sqrt{1+2 \ln [(1-\tilde{\beta}) / \tilde{\beta}] / g^{2}(\beta)}-1}{\ln [(1-\tilde{\beta}) / \tilde{\beta}]} .
$$

In the end, the precision estimate of two measurements with a 1 -fold and an $n_{2}$-fold rotation is given by

$$
\operatorname{Prob}\left[\left|\phi-\tilde{\phi}_{2}\right| \leqslant g(\beta) \sigma_{2}\right]=(1-\beta)(1-\tilde{\beta}),
$$

where $\tilde{\phi}_{2}$ is the measured and selected value of the parameter $\phi$ in the second step and the error bar is given by $\sigma_{2} \equiv \sigma_{n_{2}}=\sigma_{1} / n_{2}$. Thus the second measurement improves the precision by the large factor $\sqrt{N} v(\beta, \tilde{\beta}) \gg 1$, with $v(\beta, \tilde{\beta}) \approx 0.96$ for $\beta=\tilde{\beta}=0.01$ (for small $\beta, \tilde{\beta} \ll 1$, $v \approx(\pi \sqrt{2|\ln \beta|)} /|\ln \tilde{\beta}|)\left[(1+\ln \tilde{\beta} / \ln \beta)^{1 / 2}-1\right]$. The $K$-fold iteration of this procedure will further improve the precision of the measurement.

The above measurement protocol involves two sources of error: the estimation error $\beta$, for the angle $\phi$ to lie outside the interval $\left|\phi-\tilde{\phi}_{2}\right| \leqslant g(\beta) \sigma_{2}$; and the classification error $\tilde{\beta}$, for an incorrect choice of the alternative $A_{k}$. While an estimation error can be ruled out in a subsequent step, this is not the case for a classification error. Indeed, for $K=2$ and assuming that we have correctly identified the peak $\tilde{\phi}_{n k}=\tilde{\phi}_{2}$, let us suppose that $\left|\phi-\tilde{\phi}_{2}\right|>g(\beta) \sigma_{2}$, hence the true value $\phi$ is outside the allowed range. Applying $n_{3}=v / \sigma_{2}$ rotations in the next step, none of the peaks $\tilde{\phi}_{n_{3} k}$ will belong to the interval $\tilde{I}_{2}$ defined through $\left|\phi-\tilde{\phi}_{2}\right| \leqslant g(\beta)\left(\sigma_{2}-\sigma_{3}\right)$ with probability $1-\beta$, signaling the error in one of the previous steps. The classification error cannot be caught in subsequent steps: Consider two alternative angles $\phi$ localized either near (a) the $k$ th peak, $\left|\phi-\tilde{\phi}_{n_{2} k}\right| \leqslant g(\beta) \sigma_{2}$, or (b) the next peak, $\left|\phi-\left(\tilde{\phi}_{n_{2} k}+2 \pi / n_{2}\right)\right| \leqslant g(\beta) \sigma_{2}$. Then applying an $n_{3}$-fold rotation, the random parameter $\phi_{3}=n_{3} \phi$ will be localized within (a) $\left|\phi_{3}-n_{3} \phi_{n_{2} k}\right| \leqslant g(\beta) v$ or (b) $\mid \phi_{3}-$ $n_{3} \phi_{n_{2} k}-2 \pi v \sqrt{N} \mid \leqslant g(\beta) v$. Since these intervals are largely overlapping, hypotheses (a) and (b) cannot be distinguished.

Iterating the process $K$ times, our measurement protocol satisfies the confidence criterion

$$
\operatorname{Prob}\left[\left|\phi-\tilde{\phi}_{K}\right| \leqslant g(\beta) \sigma_{K}\right]=(1-\beta)(1-\tilde{\beta})^{K-1},
$$

with $\sigma_{K}=\sigma_{1}\left[\sigma_{1} / \nu\right]^{K-1} \sim N^{-K / 2}$. To reach a given precision $\delta$ one then needs to perform $K_{\delta} \sim 1+\ln \left(\sigma_{1} g / \delta\right) / \ln (v \sqrt{N})$ steps; at the same time, the overall confidence level decreases exponentially, $\propto \exp \left(-\tilde{\beta} K_{\delta}\right)$, with the number $K_{\delta}$ of steps. Moreover, an estimation error, i.e., none of the peaks belongs to the prescribed estimation interval, forces one to repeat the entire procedure. However, in practice, $\sqrt{N} \sim 30-40$ and thus just a few steps are required to reach a good precision $\delta$.

In counting the number of resources needed to reach the estimate, (18), we choose as our basic unit the operation $\hat{U}_{z}[\phi]$ applied to a single qubit. The $i$ th step in the above procedure requires (for $N$ qubits in the ensemble) $R_{i}=N n_{i}=N\left(v / \sigma_{1}\right)^{i-1}=v^{i-1} N^{(i+1) / 2}$ elementary operations. For $v / \sigma_{1} \gg 1$, the entire $K$-step process then involves $R=\sum_{i=1}^{K} R_{i} \approx R_{K}$ operations. Expressing the prescribed precision $\delta_{K}\left(\approx g \sigma_{K}=g \sigma_{1}\left[\sigma_{1} / \nu\right]^{K-1}\right)$ through the number $R$ 
of resources, we arrive at the scaling

$$
\delta_{K}=\frac{g(\beta)}{[v(\beta, \tilde{\beta})]^{\frac{K-1}{K+1}}} \frac{1}{R^{\frac{K}{K+1}}} \sim \frac{\delta_{0}}{N^{K / 2}},
$$

telling us that the Heisenberg limit is reached asymptotically at large values of $K$. In an actual implementation with ultracold atoms, the time needed to prepare the atomic ensemble is typically a few seconds [8], and increasing the number of rotations $n$ does not significantly increase the overall duration of the measurement. The standard statistical measurement requires $K_{\text {std }} \sim\left(\delta_{0} / \sqrt{N} \delta\right)^{2}$ preparations to reach a precision $\delta$, which is exponentially larger than the $K_{\delta}$ preparations required with the present protocol, $K_{\text {std }} \sim N^{K_{\delta}-2}$.

Dephasing. In addition to the unitary rotation $\hat{U}_{z}[\phi]$, the qubits may experience a stochastic field $\varphi(t)$, e.g., due to uncontrolled interactions between qubits generating a different phase shift $\phi \rightarrow \phi+\int d t \varphi(t)$ for each qubit. Averaging the single-qubit density matrix over $\varphi(t)$, the ofdiagonal amplitudes $\epsilon \exp ( \pm i \phi)$ are reduced by the factor $\epsilon=$ $\exp \left(-\Gamma \tau_{1} / 2\right)<1$, where we have assumed a Gaussian random field $\left\langle\varphi(t) \varphi\left(t^{\prime}\right)\right\rangle=\Gamma \delta\left(t-t^{\prime}\right)$ and $\tau_{1}$ is the exposure time of the primary measurement; the reduction in these amplitudes after an $n$-fold rotation is given by $\epsilon^{n}$. Measurement of the ensemble polarization involves the parameter $p=\epsilon^{n} \cos (n \phi)$, and the width in the a posteriori distribution function is $\sigma_{n} \rightarrow \sigma_{1} /\left(n \epsilon^{n}\right)$. The smallest attainable width, $\sigma_{1} e \ln (1 / \epsilon)$, is reached after $n_{c}=-1 / \ln \epsilon=\tau_{c} / \tau_{1}$ steps, with $\tau_{c}=2 / \Gamma$ the coherence time.

Application. We analyze the use of our protocol to measure a constant magnetic field $B$ with an atomic ensemble, considering a transition with differential magnetic moment of order $\mu_{\mathrm{B}}$, the Bohr magneton. Assuming the prior knowledge that $B<B_{+}$, we choose the interrogation time of the first Ramsey sequence $\tau_{1} \sim 2 \pi \hbar / \mu_{\mathrm{B}} B_{+}$such that the accumulated phase $\phi=\mu_{\mathrm{B}} B \tau_{1} / \hbar$ does not exceed $2 \pi$. This primary measurement results in a phase uncertainty of $[\delta \phi]_{1}=1 / \sqrt{N}$, translating to a precision of $[\Delta B]_{1}=\hbar / \mu_{\mathrm{B}} \tau_{1} \sqrt{N}$ in the field. In the following steps, the Ramsey time is increased as described above. The longest Ramsey sequence of duration $\tau_{c}=n_{c} \tau_{1}$ provides us with a precision of $[\delta \phi]_{\min }=1 / n_{c} \sqrt{N}$ for the phase estimation and, thus, a field precision of $[\Delta B]_{\min }=$ $\hbar / \mu_{\mathrm{B}} \tau_{c} \sqrt{N}$ using $K \sim 1+\ln \left(\tau_{c} / \tau_{1}\right) / \ln (\sqrt{N})$ steps.

In a realistic situation the above procedure is feasible for small magnetic fields, since the duration $\tau_{1}$ of the first Ramsey sequence cannot be arbitrarily small: A typical $\tau_{1} \sim 10^{-6} \mathrm{~s}$ corresponds to a field $B_{+} \sim 1 \mathrm{G}$. With a typical coherence time $\tau_{c} \sim 1 \mathrm{~s}$ and $N \sim 1000$ atoms one arrives, after $K=5$ steps, at a precision $\Delta B \sim 3 \times 10^{-9} \mathrm{G}$. In order to measure higher fields one can exploit the phase periodicity and subtract an offset field. This requires prior knowledge that the field lies in an interval $\left[B_{-}, B_{+}\right]$. In this case, given a minimal time $\tau_{1}$, we choose some field $B_{0} \in\left[B_{-}, B_{+}\right]$that satisfies the matching relation $\mu_{\mathrm{B}} B_{0} \tau_{1} / \hbar=2 \pi M$, with $M$ the largest possible integer. The procedure described above is then used to measure the remaining small field $b=B-B_{0}$. In an experimental implementation, instead of applying an offset field $B_{0}$, the same effect can also be obtained by adjusting the frequency of the local oscillator that drives the $\pi / 2$ pulses of the Ramsey sequence.

Conclusion. It is interesting to compare our ensemble-based algorithm with Kitaev's original phase estimation algorithm involving individual qubits. In order to reach a prescribed precision $\delta$, the latter necessitates $K \sim \ln (1 / \delta) / \ln 2$ steps, a factor $(\ln \sqrt{N}) / \ln 2$ larger than the ensemble-based protocol. For the Kitaev algorithm, the resources scale as $\delta \sim \ln R / R$ (accounting for the fact that a final error probability $\beta$ necessitates a smaller value $\beta / K$ for the individual step [12]), which is better than our algebraic relation $\delta \sim R^{-K /(K+1)}$. Still, it appears quite attractive to trade this slightly inferior performance against the improved practicality of our algorithm as compared to Kitaev's original scheme: while the latter requires the precise and individual control of qubits over many steps, our ensemble-based algorithm necessitates fewer steps and only collective manipulations and measurements on the entire qubit ensemble.

The sequential strategy discussed here is particularly useful in scanning probe measurements of a spatially varying field [9]. If the field distribution is a priori unknown, the duration of the initial Ramsey sequence has to be very short at each pixel of the image. Our scheme allows us to optimally adapt the sequence at each pixel to reach a fast reduction in the measurement uncertainty. This dramatically reduces the overall time required to record a picture with a given precision. Finally, our ensemble-based sequential strategy could also be combined with a parallel strategy using squeezed Bose-Einstein condensates.

It is instructive to analyze the origin of the quantum resources [4] exploited in the present measuring protocol. Replacing the total spin observable $\hat{S}_{z}$ with a classical rotating arrow, the same algorithm can be carried out. However, during the dynamical evolution, the rotation angle of the classical arrow will pick up a noise component growing with time $\propto \sqrt{\tau}$ due to the environmental coupling, resulting in the shot-noise scaling $\delta \propto 1 / \sqrt{\tau}$. Cooling and isolating the system will lead to a quantum arrow with unitary evolution; the error appears only at the end of the measurement process and is independent of the duration $\tau$ of the measurement itself. It is this coherent evolution over increasingly longer time scales that is exploited as a quantum resource in our protocol.

Acknowledgments. We acknowledge fruitful discussions with Gordey Lesovik, Tilman Esslinger, and Joseph Renes and financial support from the Swiss National Foundation through the NCCR QSIT.
[1] V. Giovannetti, S. Lloyd, and L. Maccone, Nature Photon. 5, 222 (2011); Science 306, 1330 (2004).

[2] D. W. Berry, M. J. W. Hall, M. Zwierz, and H. M. Wiseman, Phys. Rev. A 86, 053813 (2012); V. Giovannetti, S. Lloyd, and L. Maccone, Phys. Rev. Lett. 108, 260405 (2012).
[3] C. W. Helstrom, Quantum Detection and Estimation Theory (Academic Press, New York, 1976).

[4] V. Giovannetti, S. Lloyd, and L. Maccone, Phys. Rev. Lett. 96, 010401 (2006).

[5] C. M. Caves, Phys. Rev. D 23, 1693 (1981); D. J. Wineland, J. J. Bollinger, W. M. Itano, F. L. Moore, and D. J. Heinzen, 
Phys. Rev. A 46, R6797 (1992); M. J. Holland and K. Burnett, Phys. Rev. Lett. 71, 1355 (1993).

[6] A. Fujiwara, Phys. Rev. A 63, 042304 (2001); D. G. Fischer, H. Mack, M. A. Cirone, and M. Freyberger, ibid. 64, 022309 (2001); M. Sasaki, M. Ban, and S. M. Barnett, ibid. 66, 022308 (2002).

[7] A. Luis, Phys. Rev. A 65, 025802 (2002); T. Rudolph and L. Grover, Phys. Rev. Lett. 91, 217905 (2003); M. de Burgh and S. D. Bartlett, Phys. Rev. A 72, 042301 (2005); B. L. Higgins, D. W. Berry, S. D. Bartlett, H. M. Wiseman, and G. J. Pryde, Nature 450, 393 (2007); W. van Dam, G. M. D’Ariano, A. Ekert, C. Macchiavello, and M. Mosca, Phys. Rev. Lett. 98, 090501 (2007).

[8] C. Gross, T. Zibold, E. Nicklas, J. Estève, and M. K. Oberthaler, Nature 464, 1165 (2010); M. F. Riedel, P. Böhi, Y. Li, T. W. Hänsch, A. Sinatra, and P. Treutlein, ibid. 464, 1170 (2010).

[9] C. F. Ockeloen, R. Schmied, M. F. Riedel, and P. Treutlein, Phys. Rev. Lett. 111, 143001 (2013).

[10] A. Louchet-Chauvet, J. Appel, J. J. Renema, D. Oblak, N. Kjaergaard, and E. S. Polzik, New J. Phys. 12, 065032 (2010);
I. D. Leroux, M. H. Schleier-Smith, and V. Vuletic, Phys. Rev. Lett. 104, 250801 (2010).

[11] M. Kitagawa and M. Ueda, Phys. Rev. A 47, 5138 (1993).

[12] A. Yu. Kitaev, arXiv:quant-ph/9511026; Z. Ji, G. Wang, R. Duan, Y. Feng, and M. Ying, IEEE Trans. Inf. Theory 54, 5172 (2008).

[13] G. Giedke, J. M. Taylor, D. D’Alessandro, M. D. Lukin, and A. Imamoglu, Phys. Rev. A 74, 032316 (2006).

[14] R. S. Said, D. W. Berry, and J. Twamley, Phys. Rev. B 83, 125410 (2011).

[15] M. V. Suslov, G. B. Lesovik, and G. Blatter, Phys. Rev. A 83, 052317 (2011).

[16] R. B. Griffiths and C.-S. Niu, Phys. Rev. Lett. 76, 3228 (1996).

[17] G. Waldherr, J. Beck, P. Neumann, R. S. Said, M. Nitsche, M. L. Markham, D. J. Twitchen, J. Twamley, F. Jelezko, and J. Wrachtrup, Nature Nanotech. 7, 105 (2011); N. M. Nusran, M. Ummal Momeen, and M. V. Gurudev Dutt, ibid. 7, 109 (2011).

[18] Multiple winding of the phase $\phi$ is excluded, reflecting some prior knowledge of the unknown field; otherwise, our protocol estimates $\phi$ only modulo $2 \pi$. 